\title{
Essais préliminaires d'immunisation des zébus d'élevage extensif contre la tuberculose
}

\author{
par Y. CHENEAU (*), J.-M. BLANCOU (*) et H. SERRES (**)
}

\begin{abstract}
RESUME
La comparaison de l'immunité anti-tuberculeuse conférée au zébu par l'injection d'un vaccin vivant (B.C.G.) et de trois vaccins tués (B.C.G. mort, bacilles virulents tués par les rayons ultra-violets, bacilles virulents trypsinés) démontre la supériorité de ce dernier lors d'une épreuve par voie intraveineuse.

Cette supériorité est confirmée lors de l'emploi de ce vaccin trypsiné chez des zébus d'élevage extensif soumis à une contagion naturelle.
\end{abstract}

Comme l'ont montré VALETTE (6), BLANCOU et collab. (1), la tuberculose a une incidence élevée dans les troupeaux de zébus malgaches, puisque des réactions positives à la tuberculination sont relevées sur de très nombreux animaux, qui peuvent représenter jusqu'à 40 p. 100 de l'effectif. Nous avons déjà expliqué (1) que la méthode de tuberculination élimination, efficace dans les pays développés, ne peut pas être envisagée dans un délai prévisible. A son application les facteurs économiques élèvent un obstacle insurmontable.

Il demeure cependant nécessaire d'aborder ce grave problème. Il a été décidé de préciser, par un important programme d'expérimentations, quel crédit on pouvait accorder au projet de stratégie suivante :

- Première phase: diminution de l'incidence de la tuberculose bovine par des vaccinations annuelles n'entraînant pas d'allergie durable à la tuberculine. Ces vaccinations seraient faites en même temps que les interventions prophylactiques contre les autres maladies traditionnelles.

(*) I.E.M.V.T., Laboratoire Central de l'Elevage, B.P. $n^{0}$ 862, Tananarive, République Malgache.

(**) I.E.M.V.T., 10, rue Pierre Curie, 94700 Maisons-Alfort.
- Deuxième phase : l'incidence de la tuberculose ayant diminué suffisamment, une action sanitaire devenue économiquement possible est entreprise en vue de l'éradication.

Les premières recherches visent à établir l'efficacité que l'on peut attendre des vaccinations.

\section{TYPES DE VACCINS UTILISES}

Nous avons concurremment employé un vaccin vivant et des vaccins tués.

\section{B.C.G. vivant}

Le bacille de Calmette et Guérin, très largement employé dans la prophylaxie de la tuberculose humaine, fut aussi utilisé en matière de tuberculose bovine. Malheureusement, il sensibilise les animaux à la tuberculine et rend impossible la prophylaxie par tuberculination. Pour cette raison il n'est, depuis plusieurs décennies, guère employé chez l'animal. Son activité doit, pour se manifester au mieux, être assortie de conditions d'emploi : vaccination dès la naissance, isolement des vaches contagieuses, etc.

Dans le troupeau d'élevage extensif, ces conditions ne peuvent pas être respectées et l'em- 
ploi que nous ferons du B.C.G. sera, bien malgré nous, éloigné des conditions optimales.

Néanmoins la souche B.C.G., dont le degré d'atténuation est vraiment très élevé, nous a paru être le seul antigène vivant utilisable.

\section{Vaccins tués}

a) B.C.G. chauffé: un vaccin de ce type a été utilisé sur une grande échelle par PHAM NGOC THACH (4) au Nord Viet-Nam.

Nous avons employé du B.C.G. Iyophilisé en ampoules (100 $\mathrm{mg}$ par ampoule). Ces dernières sont placées dans une étuve à $43^{\circ}$ pendant 50 jours. Puis les bacilles sont mis en suspension en eau distillée à raison de $10 \mathrm{mg} / \mathrm{ml}$, sans adjuvant de l'immunité.

b) Vaccin chauffé puis trypsiné : la préparation de ce vaccin est celle décrite par CROWLE (2) à la seule différence que les corps bacillaires morts ne sont pas écartés du vaccin. Elle libère un antigène peptido-aminopolysaccharidique. La souche utilisée, dite «B. M. », isolée localement, est de type bovin. Elle a subi 92 passages sur milieu de Sauton. Son pouvoir pathogène n'est que légèrement atténué.

Le vaccin contient $10 \mathrm{mg} / \mathrm{ml}$ d'antigène sec en eau distillée. Il est additionné de 1 p. 100 d'alun de potassium jouant le rôle d'adjuvant.

c) Vaccin tué par les ultraviolets : ce vaccin est du type de ceux préparés par MILZER et collab. (3) et SARBER et collab. (5). La même souche que ci-dessus est utilisée. Les bacilles morts sont suspendus en eau distillée à raison de $100 \mathrm{mg} / \mathrm{ml}$, sans adjuvant.

\section{RESULTATS DES ESSAIS}

\section{Essais en station}

Ils ont été faits sur des jeunes zébus ne réagissant pas à la tuberculination, présentant tous les caractères cliniques d'une bonne santé. Les animaux vaccinés sont éprouvés entre 35 et 40 jours après la vaccination par injection intraveineuse de la souche «B.M.» virulente. Ils sont sacrifiés de 50 à 92 jours après l'épreuve et une autopsie minutieuse permet de déceler des lésions tuberculeuses, même peu étendues.
Dans tous les cas, des animaux témoins non vaccinés mais éprouvés subissent les mêmes observations.

L'étude des lésions chez les animaux vaccinés permet de déterminer un « taux de lésions » et de le comparer à celui que donnent les observations faites sur les non-vaccinés; on en déduit un « taux de protection».

La détermination de ce «taux de lésions » s'effectue par les contrôles suivants :

1. Etendue des lésions macroscopiques des deux poumons (notée de 0 à 4).

2. Etendue des lésions macroscopiques de foie (notée de 0 à 2).

3. Présence ou absence de lésions de la rate (notée 0 ou 1).

4. Présence ou absence de lésions ganglionnaires (notée 0 ou 1).

5. Présence ou absence de follicules tuberculeux sur les coupes histologiques des quatre organes précédents (noteé de 0 à 4 ).

Le bilan total de ces différentes notes est ensuite exprimé en pourcentage du maximum.

Les résultats sont groupés au tableau I.

Ils montrent que le meilleur taux de protection est obtenu avec la souche B.M. chauffée et trypsinée. Ce taux est significativement supérieur aux autres. On note que, dans les conditions de l'expérience, l'efficacité du B.C.G. vivant est très faible.

\section{Essais sur le terrain}

a) Vaccins: le vaccin chauffé et trypsiné, ainsi que le B.C.G., ont été employés pour cette expérimentation.

b) Conditions expérimentales: l'observation a intéressé 248 zébus malgaches castrés, âgés de 4 à 10 ans, se trouvant en embouche à l'herbe dans la région du Moyen-Ouest de Madagascar.

Le taux d'infection naturelle dans les troupeaux de cette région est élevé, ce qui assure une contamination d'épreuve permanente. Avant toute vaccination, les animaux sont numérotés, tuberculinés et leur réaction relevée individuellement. 
TABLEAL N $N^{\circ} \mathrm{I}$

Taux de protection observés pour les quatre vaccins

\begin{tabular}{|c|c|c|c|c|}
\hline & B.C.G. vivant & $\begin{array}{l}\text { B.C.G. tuê à } 43^{\circ} \\
\text { aluné à } 1 \text { p.100 }\end{array}$ & B.M. tuë, trypsinë & B.M. tué par rayons UV \\
\hline D.v. & $100 \mathrm{mg}$ & $100 \mathrm{mg}$ & $50 \mathrm{mg}$ & $100 \mathrm{mg}$ \\
\hline D.E. & $60 \mathrm{mg}(I . V)$. & $60 \mathrm{mg}(\mathrm{I} . \mathrm{V})$. & $100 \mathrm{mg}$ (I.V.) & $60 \mathrm{mg}$ (I.V.) \\
\hline $\mathrm{N}$ & 9 & 26 & 10 & 13 \\
\hline T.L. & $\frac{66,6}{100}$ contre $\frac{56,6}{100}$ & $\frac{42,8}{100}$ contre $\frac{68,3}{100}$ & $\frac{25}{100}$ contre $\frac{80}{100}$ & $\frac{32,1}{100}$ contre $\frac{56,2}{100}$ \\
\hline T.P. & $10 p .100(0-45)$ & 25.5 p. $100(10-42)$ & $55 \mathrm{p} \cdot 100(25-84)$ & 24,1 p. $100(5-60)$ \\
\hline
\end{tabular}

D.v. = dose vaccinala; $N$. = nombre de sujets d'expérience; T.P. I taux de protection;

D.E. = dose d'épreuve; $\mathrm{T} . \mathrm{L}$. = taux des 1 ësions; entre parenthèses : les intervalles de confiance au risque 5 p. 100.

\section{c) Vaccinations}

— Lot I : 78 animaux dont :

- 21 réagissant à la tuberculine

- 57 ne réagissant pas

sont vaccinés avec $50 \mathrm{mg}$ de vaccin trypsiné, en sous-cutanée.

- Lot II : 88 animaux dont :

- 26 réagissant à la tuberculine

- 62 ne réagissant pas

reçoivent une injection sous-cutanée de $100 \mathrm{mg}$ de B.C.G. à raison de $10^{6}$ bacilles viables par $\mathrm{mg}$.

- Lot III : 82 animaux dont :

- 28 réagissant à la tuberculine

- 54 ne réagissant pas

ne reçoivent aucune vaccination et servent de témoins.

La répartition en lots a été faite au hasard. d) Innocuité des vaccinations

La réaction à l'inoculation du B.C.G. est bien connue.

A la suite de l'injection de vaccin trypsiné apparaît sous la peau un nodule induré de 3 à $4 \mathrm{~cm}$ de diamètre. Quelques semaines plus tard, il peut s'abcéder, puis être éliminé. On n'a jamais observé de réaction générale, que les animaux réagissent préalablement ou non à la tuberculine.

Les vaccinations n'aggravent pas une tuberculose en train d'évoluer.

Le tableau II indique les lésions observées chez les bœufs ayant réagi positivement à la tuberculination, vaccinés ou non. Il apparaît que la gravité de la tuberculose est la même dans les trois lots. Par conséquent, on ne peut pas suspecter les vaccins d'aggraver la maladie.

TABLEAU $\mathrm{N}^{\bullet} \mathrm{II}$

\begin{tabular}{|c|c|c|c|}
\hline Lats & $\begin{array}{l}\text { Lot I } \\
\text { Vaccin trypsine }\end{array}$ & $\begin{array}{c}\text { Lot II } \\
\text { Vaccin B.C.G. }\end{array}$ & $\begin{array}{l}\text { Lot III } \\
\text { Témoins non vaccinés }\end{array}$ \\
\hline Catëgorie 1 & 7 & 10 & 14 \\
\hline Catégorie 2 & $?$ & 3 & 1 \\
\hline Catégorie 3 & 10 & 11 & 11 \\
\hline Catégorie 4 & 2 & 2 & 2 \\
\hline Formes contagieuses & 9 & 11 & 11 \\
\hline Formes non contagieuses & 12 & 15 & 17 \\
\hline$T \circ t$ a 1 & 21 & 26 & $2 B$ \\
\hline
\end{tabular}




\section{e) Efficacité des vaccinations}

Elle est jugée en comparant les lésions relevées sur 173 sujets ne réagissant pas à la tuberculination au début de l'expérience.

Les animaux tuberculeux, suivant les lésions observées, sont cotés de 1 à 4 selon le barème :

$1=$ complexe primaire dissocié ou non;

$2=$ complexe primaire avec dissémination à un ou plusieurs ganglions non viscéraux;
3 = complexe primaire avec dissémination à un ou plusieurs ganglions viscéraux, organes ou séreuses;

4 = tuberculose généralisée.

Les animaux sains sont cotés 0 .

Le pourcentage d'animaux infectés dans chaque lot définit un taux de contamination.

Les résultats sont rassemblés dans le tableau III.

TABLEAU $N^{\bullet}$ II I

\begin{tabular}{|c|c|c|c|}
\hline Lésions $\quad$ Lots & $\begin{array}{l}\text { Lot } \mathrm{I} \\
\text { Vaccin trypsinê }\end{array}$ & $\begin{array}{l}\text { Lot II } \\
\text { Vaccin B.c.G. }\end{array}$ & $\begin{array}{l}\text { Lot III } \\
\text { Tëmoins non vaccinés }\end{array}$ \\
\hline Catégorie 1 & 13 & 24 & 20 \\
\hline Catégorie 2 & 1 & 0 & 3 \\
\hline Catêgorie 3 & 5 & 6 & 5 \\
\hline Catëgorie 4 & 0 & 2 & 1 \\
\hline Absence de lésions & 38 & 30 & 25 \\
\hline Formes contagieuses & 6 & 9 & 9 \\
\hline Formes non contagieuses & 13 & 23 & 20 \\
\hline Effectif observe & 57 & 62 & 54 \\
\hline Taux de contamination & 33,3 p. 100 & $51,6 \mathrm{p} .100$ & 53,7 p. 100 \\
\hline
\end{tabular}

Ce tableau montre que:

1. le taux de contamination est nettement plus faible dans le lot ayant reçu une vaccination au "B.M. » trypsiné que dans ceux ayant reçu du B.C.G. ou non vaccinés, très proches l'un de l'autre. L'écart est statistiquement significatif.

2. pour ce même lot, on note l'absence de tuberculose généralisée et un nombre plus faible de cas contagieux.

\section{f) Sensibilisation à la tuberculine}

L'allergie postvaccinale, déterminée par la lecture (à la $72^{\mathrm{e}}$ heure) de la réaction à l'injection intradermique de 2.500 U.I. de tuberculine, a été suivie sur 49 bovins ne réagissant pas précédemment, après injection de vaccin trypsiné.

La proportion de sujets réagissants est la suivante :

1 mois après la vaccination .

63 p. 100

3 mois après la vaccination .
6 mois après la vaccination

20 p. 100

9 mois après la vaccination .

6 p. 100

Une observation complémentaire sur 47 autres bovins nous a montré que la désensibilisation était totale dès la $2^{*}$ année.

\section{CONCLUSION - DISCUSSION}

Dans les conditions de l'élevage extensif, il est certain que l'emploi du B.C.G. ne présente pas d'intérêt et que l'on ne peut fonder aucune prophylaxie sur son utilisation.

Par contre, un vaccin tué par la chaleur et trypsiné permet d'enregistrer, après une seule injection, une diminution significative de la contagion.

Un espoir peut être nourri d'obtenir sans danger, par une répétition des sollicitations antigéniques, une immunité d'un niveau suffisant pour que le taux d'infection des trou- 
peaux puisse notablement diminuer, ce qui nous mettrait en bonne voie de réalisation de la phase I du plan de prophylaxie que nous avons évoqué au début de cet article.

\section{SUMMARY}

First trials for the immunization of unhoused zebu cattle against tuberculosis

Immunity experiments on zebu cattle with live vaccine (B.C.G.) or killed yaccines (dead B.C.G. or virulent bacilli ultra-violet killed or heat-killed and trypsin extracted) showed that the last one give a better protection against an intra-venous challenge.

This protection is still the best in experiments on unhoused zebu cattle exposed to natural infection.

\section{RESUMEN}

Ensayos preliminares de inmunización de cebues de ganaderia extensiva contra la tuberculosis

La comparación de la inmunidad antituberculosa dada al cebù por la inyección de una vacuna viva (B.C.G.) y de tres vacunas matadas (B.C.G. muerto, bacilos virulentos matados por los rayos ultravioletas, bacilos virulentos tripsinados) demostra la superioridad de este último durante una prueba por via intravenosa.

Dicha superioridad se confirma en el momento del empleo de esta vacuna tripsinada en cebues de ganaderia extensiva sometidos a un contagio natural.

\section{BIBLIOGRAPHIE}

1. BLANCOU (J.), ROHRBACH (C.), PERDRIX (A.), CHOQUEL (P.) et ROSNER (G.). La tuberculose bovine à Madagascar. Rev. Elev. Méd. vét. Pays trop., 1971, 24 (4): 505-517.

2. CROWLE (A. J.). Immunogen extracted from tubercle bacilli with trypsin. Zeit Imm. Allerg. Klin. Immun., 1969 (1-3): 71-78.

3. MILZER (A.), LEVINSON (S. O.) et LEWIS (M. B.). Immunization of mice with ultra violet killed tuberculosis vaccines. Proc. Soc. Exp. Biol. New York, 1950, 75: 735-736.
4. PHAM NGOC THACH, NGUYEN THI HEI, DANE DUC TRACH, TANG HUU THANH et NGUYEN DINH HUONG. L'immunisation contre la tuberculose par le B.C.G. tué. Rev. immunol. Thér. antimicrob., Paris, 1968, 32 (1-2): 77-90.

5. SARBER (R. W.), RUNGESTER (W. J.) et STIMPERT (F. D.). Immunization studies with irradiated tuberculosis vaccines. Ann. Rev. Tub., 1950, 62 : 418-427.

6. VALETTE (P.). La tuberculose animale à Madagascar. - Tananarive, Direction de l'Elevage, 1971. $58 \mathrm{p}$. 